\title{
Misfit dislocation superlattices in IV-VI multilayered compounds as zero-dimensional quantum boxes
}

\author{
A. Yu. Sipatov \\ Kharkov State Polytechnic University, 21 Frunze St., Kharkov, 310002, Ukraine \\ E-mail: sipat@kpi.kharkov.ua \\ Received Jule 20, 1998, revised December 8, 1998
}

\begin{abstract}
Quantum dots can be generated by a new method based on the formation of misfit dislocations in a perfectly periodic array network during the epitaxial growth of IV-VI compound superlattices. Infrared photoluminescence spectra of IV-VI superlattices are measured at temperatures $T=5-90 \mathrm{~K}$. A large blue shift and temperature independent line positions and width were observed in $\mathrm{PbSe}-\mathrm{PbS} /(001) \mathrm{KCl}$ superlattices with a three-dimensional periodic arrays of misfit dislocations. It is supposed that these features are due to quantum dot luminescence.
\end{abstract}

PACS: 68.65.+g, 78.55.-m

\section{Introduction}

Any new attempt to create quantum dots is great importance because, compared with $2 D$-structures, it is more difficult to build up both $0 D$ and $1 D$ objects. Recently a new way has been found to create quantum dots of InAs with help of self-organizing processes in Stranski-Krastanov island growth during molecular beam epitaxy on a GaAs substrate [1]. Another example is the creation of $1 D$ wires by dislocation slipping induced reduction in dimensionlity of $2 D$ structure down to one dimension [2]. The present contribution is devoted to the investigation of the photoluminescence spectra of IV-VI superlattices with features due to lattice misfit dislocation. These superlattices reveal the specific properties of $0 D$ systems such as a remarkable blue shift and temperature independent line positions and width. Thus it is a new way of creating quantum dots in IV-VI superlattices via misfit dislocation generation during the epitaxial growth process. It is known that there exists a nearly ideal periodic grid of interface misfit dislocations (MD) in strained IV-VI superlattices [3,4]. The lead and rare-earth chalcogenides are quite suitable for preparation of such misfit dislocation superlattices. These materials have NaCl-crystal structure, and provide a wide range of lattice misfit $(f=0.5-13 \%)$ as well as of misfit dislocation periods $(D=3-50 \mathrm{~nm})$.

\section{Sample characterization}

The multilayers were prepared by thermal or electron beam evaporation of lead and rare-earth chalcogenides in vacuum $\left(10^{-4}-10^{-5} \mathrm{~Pa}\right)$ and alternate deposition of two compounds onto a $(001) \mathrm{KCl}$ substrate at the temperature $500-550 \mathrm{~K}$. The layer thickness and the growth rate were controlled by quartz resonator. Superlattices with periods $5-500 \mathrm{~nm}$ and number of periods $N=2-50$ were grown. Structure investigation by X-ray diffraction (XRD) and transmission electron microscopy (TEM) shows that growth in $\langle 001\rangle$ direction is going on to the layer-by-layer (Frank-van der Merwe) mechanism and square grids of misfit dislocations are formed at the interfaces with a period $D=3-50 \mathrm{~nm}$. The MD grid periodicity is much better for a sufficienlty large layer lattice misfit $(f>2 \%)$. Such MD grid may be considered as a new kind of superlattice misfit dislocation superlattice (MDSL). The MDSL is the 3-dimensional superlattice with periodic modulation by misfit dislocation arrays in the plane of multilayer structure and composition modulation along growth direction. The MDSL of above type with multilayers of $\mathrm{PbSe}-\mathrm{PbS}$ was prepared by thermal evaporation and alternate deposition of $\mathrm{PbSe}$ and $\mathrm{PbS}$ on $(001) \mathrm{KCl}$ substrate. The specimen has 40 layers, each $7.0 \mathrm{~nm}$ in thickness, and $\mathrm{MD}$ periodic grids with $D=13.5 \mathrm{~nm}$. The structural properties were checked by XRD and TEM. X-ray 


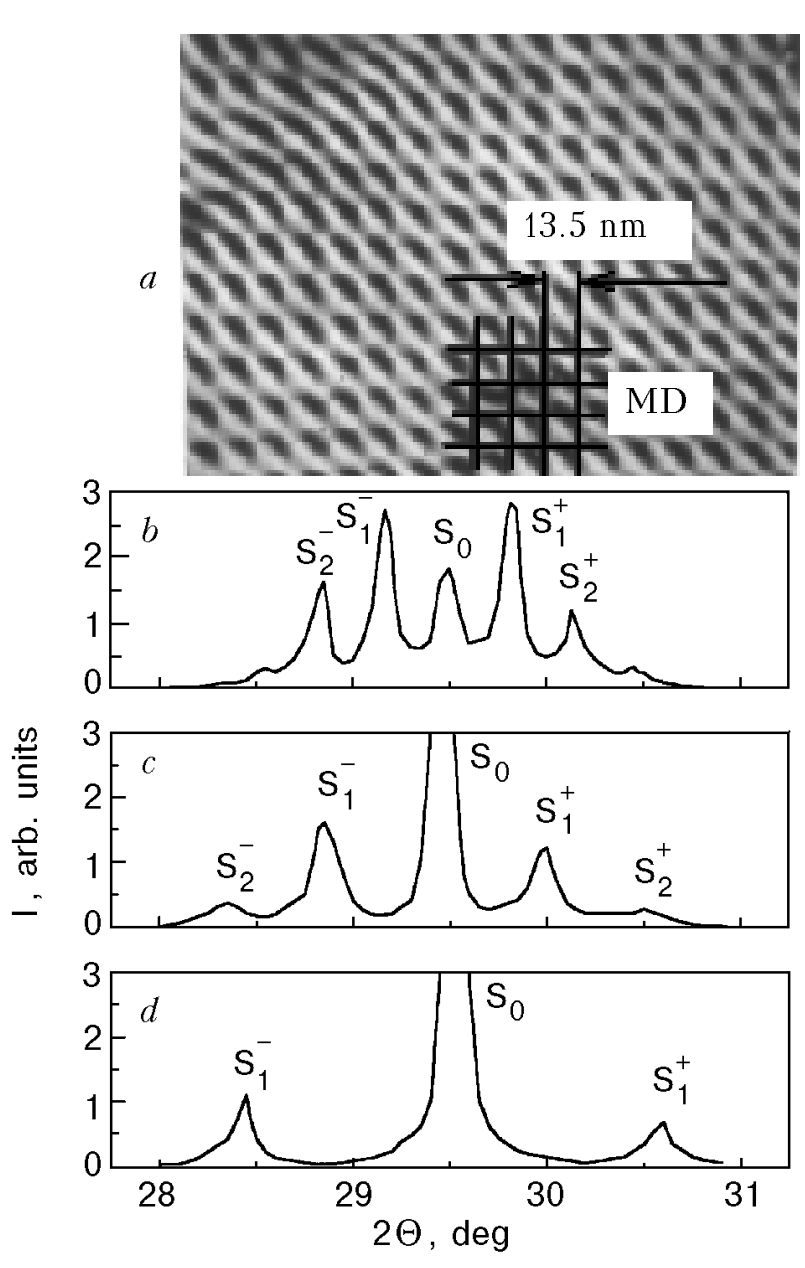

Fig. 1. Electron-microscope image of two-layer $\mathrm{PbSe}-\mathrm{PbS}$ film (a) with thickness of PbSe and PbS equal to $30 \mathrm{~nm}$ and (200) $\mathrm{X}$-ray diffraction pattern of $\mathrm{PbSe}-\mathrm{PbS}$ superlattices with periods $D_{S L}=28 \mathrm{~nm}(b), 15.8 \mathrm{~nm}(c), 8.1 \mathrm{~nm}(d) . S_{n}$ - satellite reflectioins, MD - misfit dislocations.

diffraction investigations supported the evidence of periodicity along the superlattice growth direction and where a TEM proved the existence of high quality inplane MD grids and thus gave evidence for further dimen-sion reduction. The electron $\mathrm{mi}-$ croscope image of two-layer $\mathrm{PbSe}-\mathrm{PbS}$ film with thickness $\mathrm{PbSe}-30 \mathrm{~nm}$ and $\mathrm{PbS}-30 \mathrm{~nm}$ is shown in Fig. 1,a. X-ray diffraction patterns of $\mathrm{PbSe}-\mathrm{PbS}$ superlattices with different periods are shown in Fig. 1,b-d.

\section{Results of measurements and discussion}

Photoluminescence spectra of $\mathrm{PbSe}-\mathrm{PbS}$ superlattices were earlier discussed in Ref. 5 for $T=80 \mathrm{~K}$ where two-dimensional size quantizing effects were observed (blue shift in photoluminescence spectra). In the above paper only $\mathrm{PbSe}-\mathrm{PbS} /(111)$ superlattices without misfit dislocations were investigated. In the present work the infrared photoluminescence spectra at $T=5-90 \mathrm{~K}$ in (001)-oriented superlattices with misfit dislocation grids are investigated

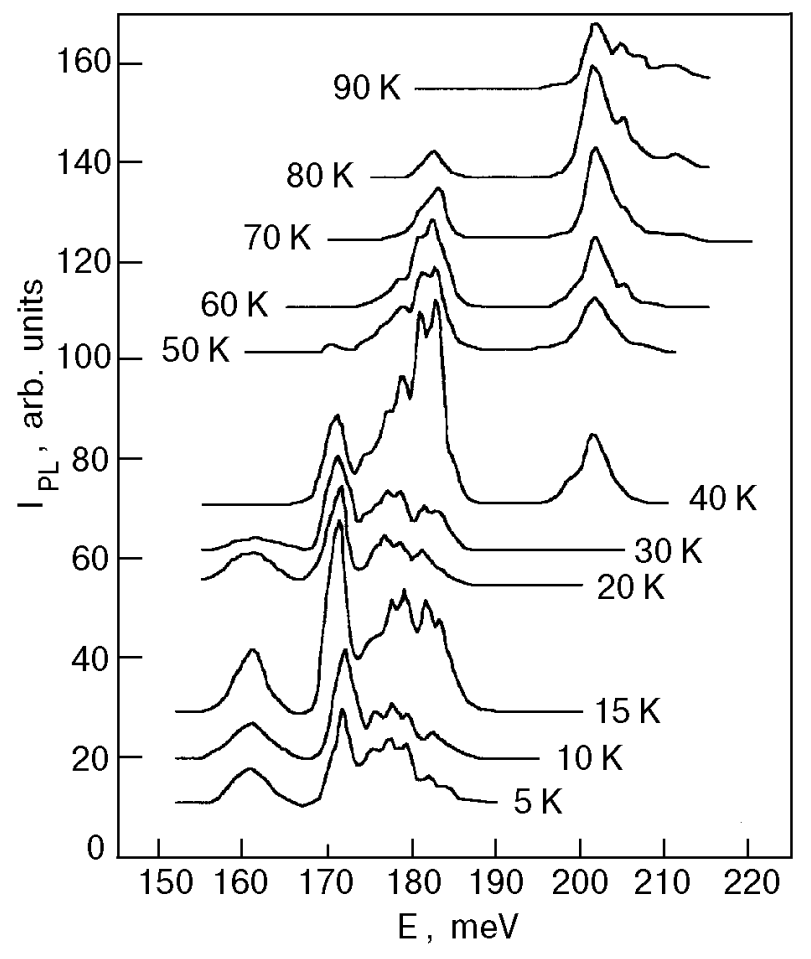

Fig. 2. Photoluminescence emission spectra of $\mathrm{PbSe}-\mathrm{PbS}$ superlattice at temperature $T=5-90 \mathrm{~K}$. The superlattice period $D_{S L}=d_{\mathrm{PbSe}}+d_{\mathrm{PbS}}=14 \mathrm{~nm}\left(d_{\mathrm{PbSe}}=d_{\mathrm{PbS}}\right)$, the number of periods -20 .

and the results are shown in Fig. 2. There are four main lines, whose positions and widths do not depend on temperature for the temperature range in which they exist. A MDSL has a compositional modulation along the plane normal to superlattice and additionally a deformation induced, band gap modulation by periodic MD arrays in the plane. The MD stresses have been calculated using methodics [4] and it appears that the amplitude of the oscillations of the MD stresses near the interface are as high as $10^{9} \mathrm{~Pa}$ (Fig. 3,a). The local position dependence of the energy gap is schematically shown in Fig. $3, b$. The coordinates $(x, y)$ demote the position in the interface plane and in the perpendicular direction, respectively. One can see from this picture that lattice deformation and gap modulation decrease with distance from the interface $y$. The strain variation of the band gap width $\Delta E_{g}=$ $=(\delta E / \delta P) \sigma_{11}$, where $(\delta E / \delta P)$ for PbSe equals $8 \cdot 10^{-11} \mathrm{eV} / \mathrm{Pa}$ [6]. From the above estimation it is clear that if we consider the real values of normal strain $\sigma_{11}$ near interface PbSe-PbS (Fig. 3,a) the variation in the magnitude of the band gap width $\Delta E_{g}$ PbSe (Fig. 3,b) can achieve 30\%-15\% for distance from interface $1 \mathrm{~nm}$ and $2 \mathrm{~nm}$, respectively. That is why the modulation of the energy gap is deep enough to form a quantum well in the interface plane and confines particles in $0 D$ quan- 


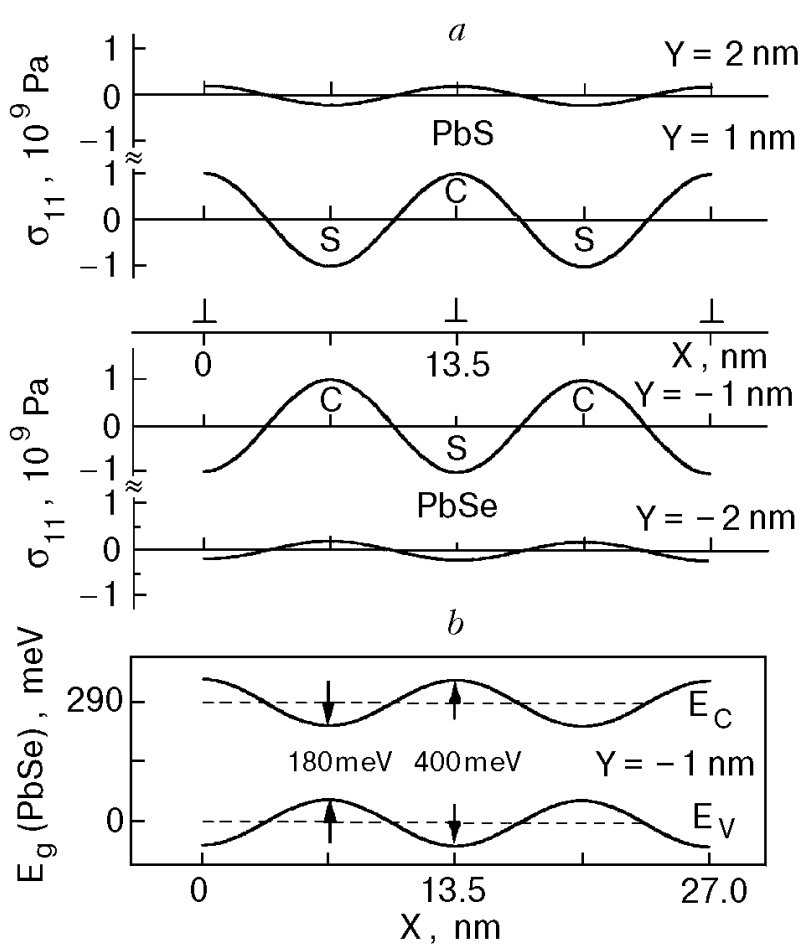

Fig. 3. Normal stress $\sigma_{11}$ distribution (a) in two-layer PbSe$\mathrm{PbS}$ film near the interface and PbSe energy band gap modulation (b) by misfit dislocation stresses at $300 \mathrm{~K}$. Y - distance from $\mathrm{PbSe}-\mathrm{PbS}$ interface; $\perp-$ position of the misfit dislocation; C - compressed region; $\mathrm{S}-$ stretch region.

tum boxes. It is very important that, for a certain sign of the misfit in $\mathrm{PbSe}-\mathrm{PbS}$ interfaces, the deformation-changed PbSe energy gap has minima in between two dislocation lines that are separated by a distance of $13.5 \mathrm{~nm}$. It means that each optically active $\mathrm{PbSe}$ region, bounded by four $\mathrm{MD}$ planes and two interface planes, is free of traps that are placed close along to dislocation lines and thus the properties are sufficient for photoluminescence signal observation. The blue shifts of reference $E_{g}(T)$ on $15-30 \mathrm{meV}$ were observed in the emission spectra of superlattices in Fig. $2\left(E_{g}=145 \mathrm{meV}\right.$ for bulk unstrained $\mathrm{PbSe}$ at $T=5-10 \mathrm{~K}$ ). The positions as well as the width of the four lines observed in luminescence spectra do not depend on temperature, which is probably due to the $0 D$ size quantization of the PbSe quantum boxes. In the model of quantum dots [7] there exist four «intraband» dipole transitions which change only the state of one particle in the electron-hole pairs. The energy of such transi- tions should not depend on the band gap $E_{g}(T)$ and consequently on the temperature. It is reasonable to suppose that the width of the lines is caused mainly by quantum dot size fluctuations and does not depend on temperature. A temperature increase leads to more effective phonon-induced suppression of photoluminescence such that low-lying excitations have not been observed in the emission spectra at high temperature (Fig. 2). Thus it is possible to consider the epitaxial growth induced dislocation superlattices as an interesting new object of extremely reduced dimensionality. The MDSL described above require further investigation to make clear not only the optical properties but the set of special properties which have been observed as, for instance, unusual high $T_{C}$-like superconductivity in IV-VI MDSL [8-10].

The author wishes to thank K. M. Herrmann and J. W. Tomm from Humboldt University of Berlin for help in photoluminescence measurements.

1. D. Bimberg, M. Grundmann, N. N. Ledentsov, S. S. Ruvimov, P. Werner, U. Richter, U. Gosele, J. Heydenreich, V. M Uctinov, A. Yu. Egorov, A. E. Zhukov, P. S. Kop'ev, and Zh. I. Alferov, Abstracts of Workshop on $M B E$ Growth Physics and Technology, Warsaw, Poland (1994), p. 12.

2. C. Guasch, F. Voillot, M. Goiran, J. P. Peyrade, E. Bedel, C. Fontaine, H. Atmani, and A. Rocher, Solid State Electronics 37, 567 (1994).

3. L. S. Palatnik and A. I. Fedorenko, J. Crystal Growth 52, 917 (1981)

4. I. F. Michailov, B. A. Savitskii, A. Yu. Sipatov, A. I. Fedorenko, and L. P. Shpakovskaya, Fiz. Tverd. Tela 25, 1166 (1983).

5. I. V. Kolesnikov, A. N. Kovalev, A. Yu. Sipatov, V. I. Paramonov, A. I. Fedorenko, and A. E. Yunovich, Fiz. Tekh. Poluprovodn. 23, 960 (1989).

6. Yu. I. Ravich, B. A. Efimova, and I. A. Smirnov, Investigation Methods of Semiconductors Applied to Lead Chalcogenides $\mathrm{PbTe}, \mathrm{PbSe}, \mathrm{PbS}$, Nauka, Moscow (1968).

7. H. Haung and S. W. Koch, Quantum Theory of the Optical and Electronic Properties of Semiconductors, World Scientific, Singapure (1990).

8. O. A. Mironov, O. N. Makarovskii, A. I. Fedorenko, A. Yu. Sipatov, O. N. Nashchekina, I. M. Zaritskii, and A. A. Konchits, Acta Physica Polonica A85, 603 (1994).

9. N. Ya. Fogel, A. Yu. Sipatov, A. I. Fedorenko, V. N. Rybalchenko, and V. G. Cherkasova, Fiz. Nizk. Temp. 20, 1142 (1994).

10. N. Ya. Fogel, V. G. Cherkasova, A. S. Pokhila, A. Yu. Sipatov, and A. I. Fedorenko, Czech. J. Phys. 46, 727 (1996). 